\title{
ALCOHOL RELATED COGNITIVE IMPAIRMENTS - A TYPICAL OR ATYPICAL CASE REPORT?
}

Luísa Gil ${ }^{1}$, Mariana Lázaro¹, Sónia Ferreira², Paula Diegues², Joana Teixeira², Teresa Mota²

${ }^{1}$ Centro Hospitalar Psiquiátrico de Lisboa, first authors ${ }^{2}$ Serviço Alcoologia e Novas Dependências, Centro Hospitalar Psiquiátrico de Lisboa

\section{OBJECTIVE}

Theme review on the available evidence regarding the alcohol-related cognitive impairment.

Case report presentation about a patient with Alcohol Use Disorder (AUD), cognitive complaints and comorbid medical conditions.

\section{BACKGROUND AND AIMS}

AUD has long been associated with cognitive deficits in multiple domains including visuospatial abilities, processing memory, executive functioning, attention, impulsivity, and verbal fluency. About half of the patients with AUD exhibit cognitive deficits that can influence their treatment compliance and everyday functioning. Take into account the cognitive dimension of alcohol-dependent patients is crucial for a tailored intervention.

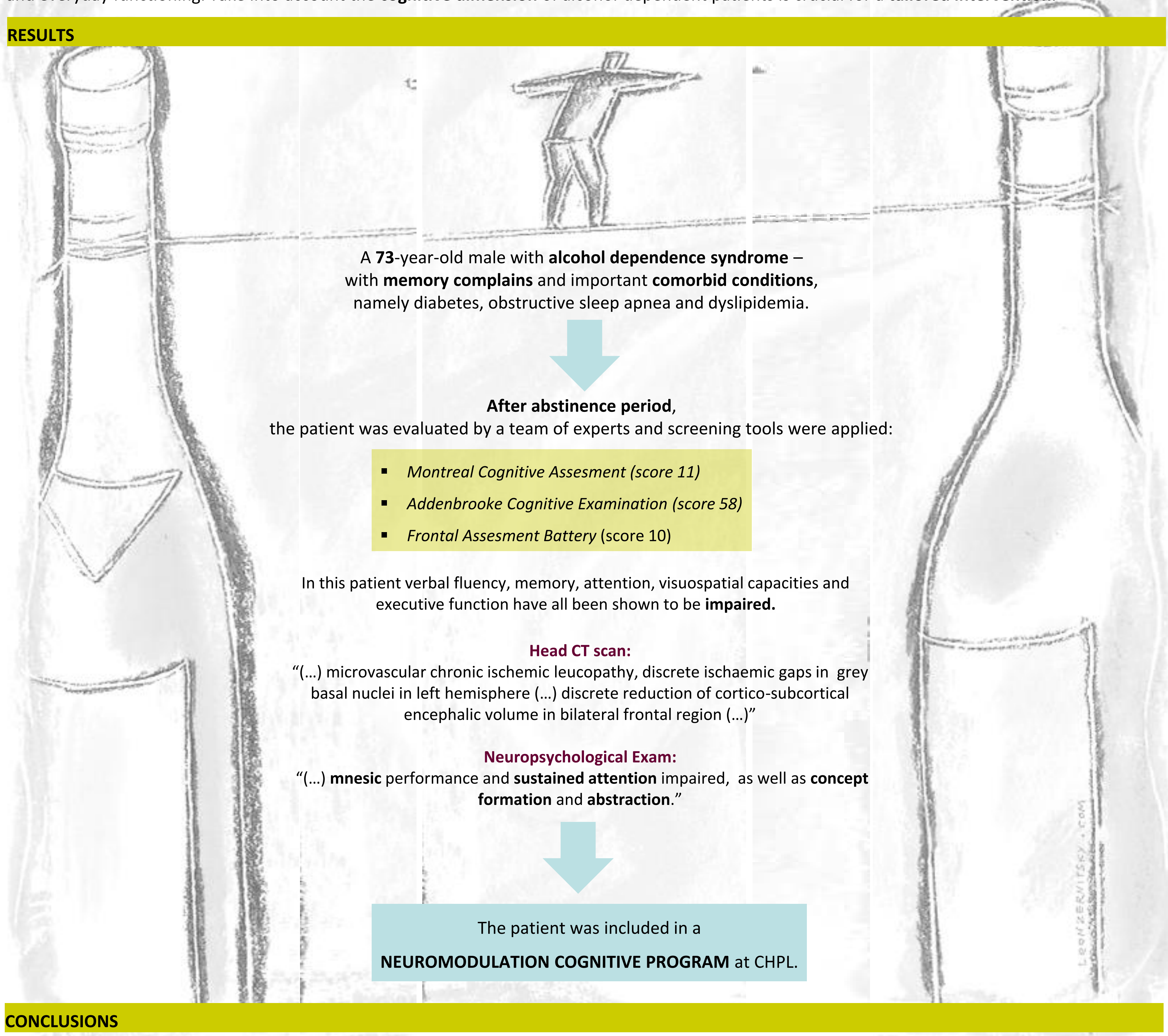

- This case report highlights the neurocognitive effect of excessive and long-term alcohol consumption and medical comorbidities.

- These cognitive impairments not only determine everyday management of these patients, but also impact on the efficacy of management and may compromise the abstinence prognosis. In fact, the therapeutical intervention requires several cognitive domains that are often impaired in theses patients, namely

\footnotetext{
- behavioral inhibitory control and cognitive flexibility to inhibit and replace the maladaptive behaviors with others that will allow the individual to lead a more fulfilling lifestyle;

- good decision-making and planning skills for appropriate prioritization of goals, instead of immediate reward;

- working memory that facilitates comprehension, reasoning, planning and problem-solving.
}

- Maintenance of lasting abstinence is associated with cognitive recovery in these patients, but some impairments may persist. In this context, recognizing and screening for alcohol-related cognitive impairments is crucial in order to facilitate treatment process and improve AUD treatment outcomes by directly promoting cognitive recovery. 